\title{
The DESI wide field corrector optics
}

\author{
Peter Doel ${ }^{\mathrm{a}}$, Michael J. Sholl ${ }^{\mathrm{b}}$, Ming Liang ${ }^{\mathrm{c}}$, David Brooks ${ }^{\mathrm{a}}$, Brenna Flaugher ${ }^{\mathrm{d}}$, Gaston Gutierrez ${ }^{\mathrm{d}}$, \\ Stephen Kent ${ }^{\mathrm{d}}$, Michael Lampton ${ }^{\mathrm{b}}$, Timothy Miller ${ }^{\mathrm{b}}$, David Sprayberry ${ }^{\mathrm{c}}$ \\ aUniversity College London, Dept. of Physics and Astronomy, Gower Street, London, UK WC1E \\ 6BT; 'bawrence Berkeley National Laboratory, 1 Cyclotron Road, Berkeley, CA, USA 94720-8141; \\ 'National Optical Astronomy Observatory, 950 North Cherry Ave., Tucson, AZ, USA 85719; Fermi \\ National Accelerator Laboratory, PO Box 500, Batavia, IL USA 60510-5011;
}

\begin{abstract}
The Dark Energy Spectroscopic instrument (DESI) is a 5000 fiber multi-object spectrometer system under development for installation on the National Optical Astronomy Observatory (NOAO) Kitt Peak 4m telescope (the Mayall telescope). DESI is designed to perform a $14,000^{\circ}$ (square) galaxy and Quasi-Stellar Object (QSO) redshift survey to improve estimates of the dark energy equation of state. The survey design imposes numerous constraints on a prime focus corrector design, including field of view, geometrical blur, stability, fiber injection efficiency, zenith angle, mass and cost. The DESI baseline wide-field optical design described herein provides a $3.2^{\circ}$ diameter field of view with six $0.8-$ $1.14 \mathrm{~m}$ diameter lenses and an integral atmospheric dispersion compensator.
\end{abstract}

Keywords: Dark Energy, Wide field corrector, Baryon Acoustic Oscillation

\section{INTRODUCTION}

The DESI project is a spectroscopic survey that has the aim of obtaining spectra for at least 18 million emission-line galaxies, 4 million luminous red galaxies and 3 million quasi-stellar objects. The primary scientific goal is to probe the origin of cosmic acceleration by employing the baryon acoustic oscillation (BAO) technique as a "standard ruler" to measure the expansion history of the Universe with improved precision, extending the measurement back to redshifts of $\mathrm{z}$ 3.7. To achieve this a new 5000 fiber multi-object spectrometer system (a schematic of which is shown in figure 1) will be constructed for installation on the 3.8m Mayall telescope at the National Optical Astronomy Observatory (NOAO) at Kitt Peak. DESI is designed to perform a 14,000 (square) galaxy and Quasi-Stellar Object (QSO) redshift survey to improve estimates of the dark energy equation of state. DESI development is expected take roughly four years, and be on-sky by early 2019.

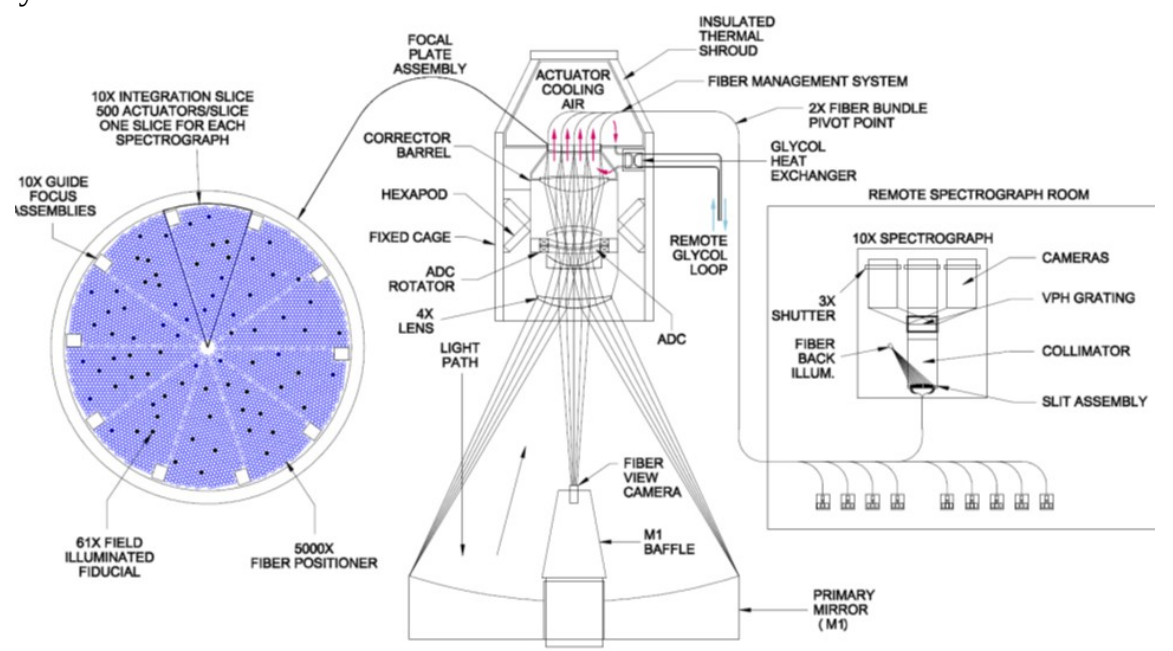

Figure 1. Schematic of the DESI instrument at the Mayall telescope 
The DESI multi-object spectrometer uses the existing primary mirror at the Mayall telescope, with a newly developed prime focus wide field corrector that allows 5,000 robotically positioned fibers to be arranged precisely over a field of view of approximately 8 square degrees.. The DESI wide field corrector will replace the existing prime focus corrector [2] at the telescope. This new corrector will consist of 6 lenses of diameters of between $0.8-1.14 \mathrm{~m}$. Similar sized correctors are operating successfully on existing telescopes, for example, the DECam instrument on the Blanco telescope [3] and the Hyper-Suprime camera on the Subaru telescope [4]. This paper focuses on the requirements placed upon the corrector, the optical design and its performance, fabrication and assembly plans, and current procurement status

\section{DESI CORRECTOR}

\subsection{Design requirements}

Development of a wide-field optical corrector for a multi-object spectrometer presents numerous singular challenges. Unlike a broad-band imaging corrector (as employed on DECam, and to be employed on LSST), the DESI corrector must correct lateral chromatic aberrations over the entire band of interest (360-980nm), in order to inject light into the $1.45 "$ diameter science fibers. Survey requirements call for observations at zenith angles of up to $60^{\circ}$, and light is much more strongly refracted in the blue than red. This introduces atmospheric dispersion which stretches the PSF toward the local horizon by several arcseconds. Figure 2 shows the atmospheric dispersion of a uniform spectrum from 360-980nm at a zenith angle of $60^{\circ}$.

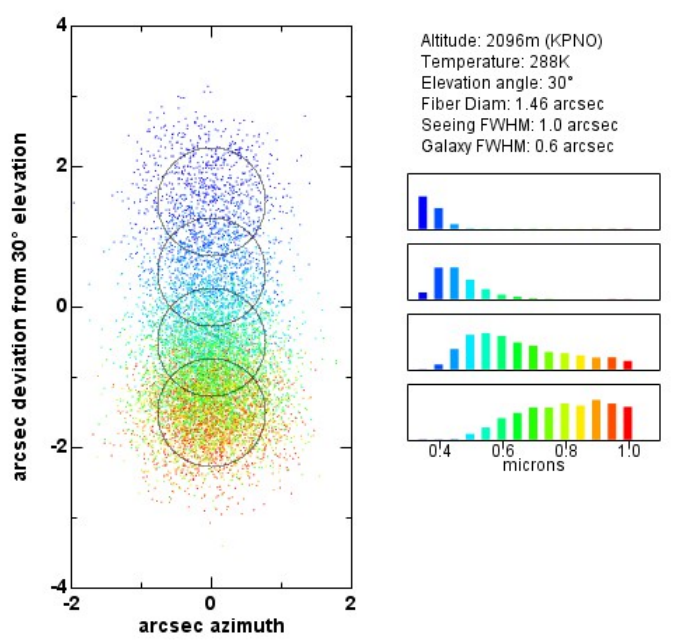

Figure 2: At a zenith angle of $60^{\circ}$, a uniform spectrum from 360-980nm is dispersed over several fiber diameters by the $1 / \lambda^{4}$ dispersion of the atmosphere. Characterization of the Ly- $\alpha$ forest, as well as identification of the Balmer break feature of LRGs requires a continuous spectrum in the blue, and requires the use of an atmospheric dispersion compensator to place light in a fiber.

A detailed analysis of the science requirements along with realistic design constraints has led to the performance and design requirements for the prime focus corrector shown in Table 1. Note that the wavelength band is divided into two sub-bands for the blur requirements, because different resolution is required for desired targets that emit at different wavelengths. 
Table 1. Performance and design requirements for the DESI instrument

\begin{tabular}{|c|c|c|}
\hline Requirement & \multicolumn{2}{|l|}{ Value } \\
\hline Wavelength band & \multicolumn{2}{|l|}{$360-980 \mathrm{~nm}$} \\
\hline $\begin{array}{l}\text { Design residual blur } \\
\text { (FWHM, arcsec) }\end{array}$ & $\begin{array}{l}\text { Zenith: } \\
\text { 360-450nm: }<0.4 \text { mean, } 0.6 \text { max } \\
\text { 450-980nm: }<0.4 \text { mean, } 0.5 \text { max }\end{array}$ & $\begin{array}{l}\text { 60deg declination: } \\
\text { 360-450nm: } 0.4 \text { mean, } 0.75 \max \\
\text { 450-980nm: } 0.4 \text { mean, } 0.6 \max \end{array}$ \\
\hline As-built corrector blur & $\begin{array}{l}\text { Zenith: } \\
\text { 360-450nm: } 0.45 \text { mean, } 0.70 \text { max } \\
\text { 450-980nm: } 0.45 \text { mean, } 0.70 \text { max }\end{array}$ & $\begin{array}{l}\text { 60deg declination } \\
\text { 360-450nm: } 0.50 \text { mean, } 0.75 \max \\
\text { 450-980nm: } 0.50 \text { mean, } 0.75 \text { max }\end{array}$ \\
\hline Field of view & \multicolumn{2}{|l|}{$33^{\circ}$ diameter } \\
\hline Focal plate diameter & \multicolumn{2}{|l|}{$812 \mathrm{~mm}$ diameter } \\
\hline Focal plate curvature & \multicolumn{2}{|c|}{ Radius of curvature (convex), greater than $3000 \mathrm{~mm}$} \\
\hline Aspheric departure slope & \multicolumn{2}{|l|}{ k30mrad } \\
\hline Chief ray deviation & \multicolumn{2}{|l|}{$<0.5^{\circ}$ average, $<1.0^{\circ}$ maximum } \\
\hline
\end{tabular}

\subsection{Optical Design}

The DESI corrector is designed to work with the Mayall 3.8m primary mirror (M1). Optical details of the Mayall M1 are shown in Table 2.

Table 2: Parameters of the Mayall 3.8m telescope primary mirror

\begin{tabular}{|c|c|}
\hline Parameter & Value \\
\hline Clear Aperture & $\varnothing 3.797 \mathrm{~m}$ \\
\hline Radius of Curvature & $21.336 \mathrm{~m}$ \\
\hline Conic Constant $(\mathrm{K})$ & -1.09763 \\
\hline
\end{tabular}

An optical design (termed Echo22) has been developed to meet the requirements shown in table 1 and a schematic of the optical layout is shown in figure 3. An atmospheric dispersion compensator (ADC) is included in the design in order to meet blur requirements at off-zenith angles. The design consists of four large fused silica elements and the ADC consisting of two monolithic N-BK7 (or S-BSL7) elements that have wedged spherical surfaces. The largest lens, C1, is $1.15 \mathrm{~m}$ in diameter and the total mass of the lenses in the design is $863 \mathrm{~kg}$. The two smaller fused silica lenses have aspheric surfaces on one face, with all other silica surfaces spherical. The lens parameters are shown in table 3.

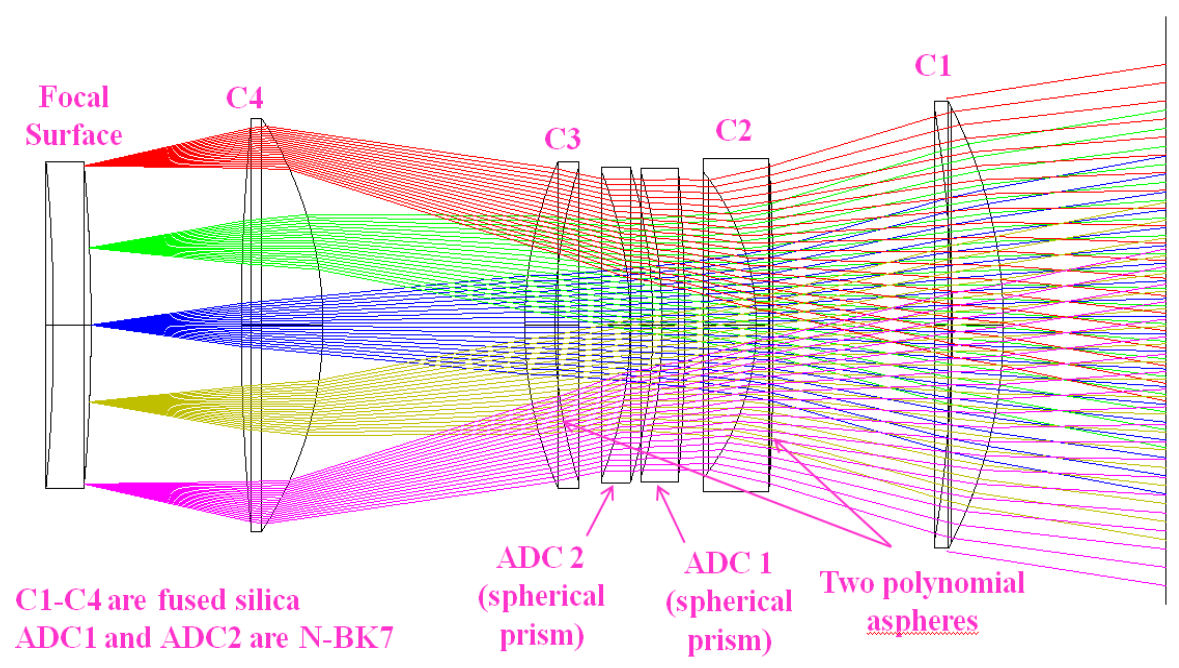

Figure 3. Schematic of the DESI optical corrector design (M. Liang, NOAO) 
The f-number of the system is 3.67 with a focal length of $13924.5 \mathrm{~mm}$. The focal plane is an aspheric surface with a radii of $4978 \mathrm{~mm}$ and a plate scale of $0.068 \mathrm{~mm} /$ arcsecond.

Table 3: DESI lens parameters

\begin{tabular}{|c|c|c|c|c|c|}
\hline Lens & $\begin{array}{c}\text { Radii } \\
\text { Front/Back } \\
(\mathrm{mm})\end{array}$ & $\begin{array}{c}\text { Center } \\
\text { Thickness } \\
(\mathrm{mm})\end{array}$ & Material & $\begin{array}{c}\text { Diameter } \\
(\mathrm{mm})\end{array}$ & $\begin{array}{c}\text { Weight } \\
(\mathrm{Kg})\end{array}$ \\
\hline C1 & $1184.8 / 3295.6$ & 136.4 & Silica & 1140 & 201 \\
\hline C2 & $12626.6 * / 612.4$ & 45.0 & Silica & 883.7 & 150 \\
\hline ADC1 & $4589.2 / 1371.0$ & 60.0 & N-BK7 & 800 & 102 \\
\hline ADC2 & $1392.1 / 1049.5$ & 60 & N-BK7 & 804 & 89 \\
\hline C3 & $-1340.7 * /-1027.0$ & 80.0 & Silica & 834 & 83 \\
\hline C4 & $934.1 / 5187.2$ & 216.9 & Silica & 1055.5 & 237 \\
\hline
\end{tabular}

*aspheric surfaces

The pointing direction of the telescope is allowed to vary slightly with zenith angle, and this allows the simpler, monolithic ADC elements. Elimination of the bonded joints on the $\sim 0.8 \mathrm{~m}$ diameter ADC prisms significantly reduces system complexity and risk. Differences in the Abbe number of the fused silica lenses and the N-BK7 ADC elements allow chromatic correction.he ADC elements have a slight $\left(\sim 0.25^{\circ}\right)$ wedge angle, which allows the user to cancel (to first order) the off-zenith dispersion of the atmosphere. The net dispersion magnitude and direction is set by rotating the ADC element when the wedges are opposed, their chromatisms subtract for net zero dispersion. If the wedges are parallel, their chromatisms add and are maximized. Wedges may be rotated in unison to change the direction of dispersion (required for an equatorial mount telescope) or separately to select the required level of dispersion for a given zenith angle. Due to the wedged, spherical ADC elements, the system has no optical symmetries. Blur distributions cannot be represented as simple radial distributions, but must be predicted over the entire focal surface.

Using the experience from the manufacture of the DECam corrector, and from discussions with potential vendors, to ease manufacture the slope of the two aspheric surfaces was constrained to be below $30 \mu \mathrm{m} / \mathrm{mm}$. In the Echo22 design the peak aspheric slope departures of lenses C2 and C3 are $<15 \mu \mathrm{m} / \mathrm{mm}$ and $<11 \mu \mathrm{m} / \mathrm{mm}$ respectively, and are shown in figure 4.
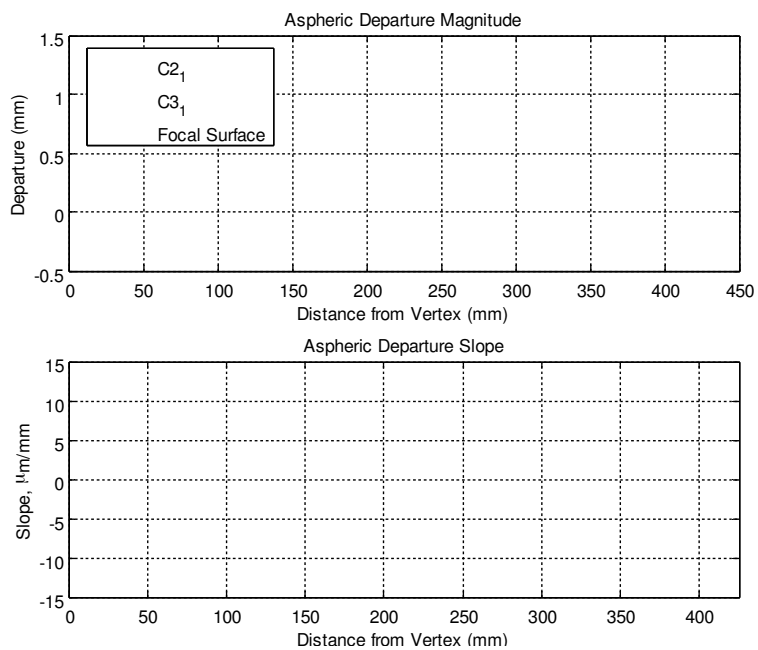

Figure 4: Aspheric departure and slope departure of asphere from best-fit sphere

The DESI optics will be coated with hard multilayer anti-reflection coatings to maximize the throughput of the system and reduce ghosting effects. 


\subsection{Imaging Performance}

Geometric blur is shown in figure 5 as a function of field and zenith angle. This is the design imaging performance of the Echo22 optical prescription (no manufacturing errors). Note the asymmetries in the blur distribution due to the ADC prisms.

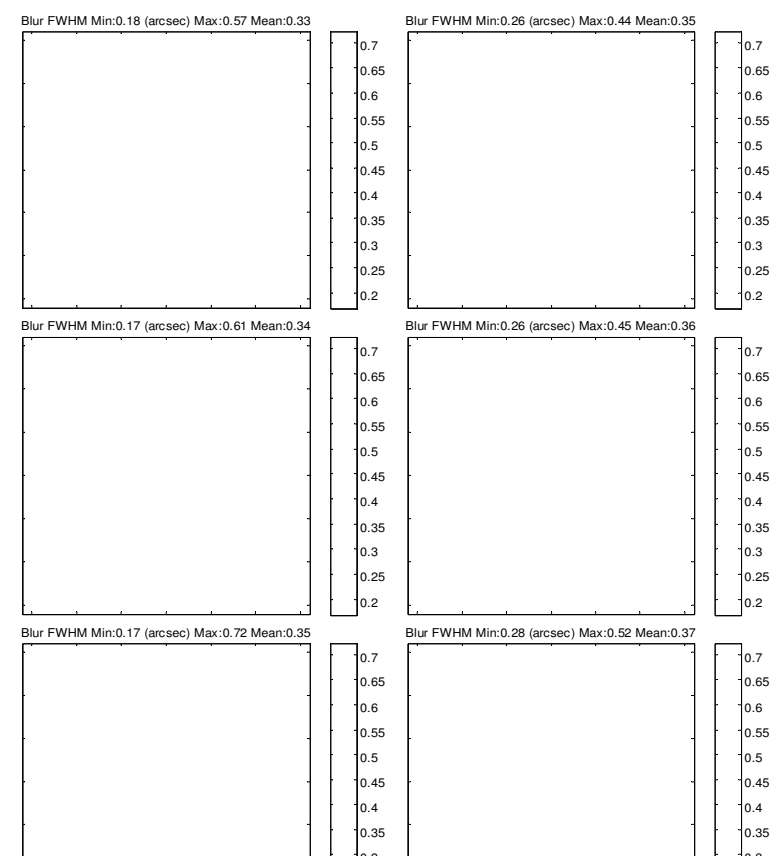

Figure 5: Geometric blur of the E22 corrector is shown at three zenith angles and two wavelength bands 360-450nm (left) and 450980nm (right). Top figures: zenith, Middle figures: $30^{\circ}$ zenith Angle, Bottom figures: $60^{\circ}$ zenith Angle

At zenith the predicted optical performance is a mean FWHM of 0.33" (max 0.57”) at a wavelength of 360-450nm, and a mean of 0.35 " (0.44" max) at a wavelength of $450-980 \mathrm{~nm}$. At a zenith angle of $60^{\circ}$ the predicted optical performance is 0.3 ” mean (max 0.72”) and at a wavelength of $360-450 \mathrm{~nm}$, and 0.37 ” mean $(0.52$ ” max) at a wavelength of $450-980 \mathrm{~nm}$.

\subsection{Field of view}

The requirement for the DESI instrument shown in table 1 was a field of view with a diameter of at least 3 degrees. The location of the prime focus corrector creates a central obscuration. The current estimate of this obscuration is Ø1.8m and coupled with the finite length of the corrector this produces a field-dependent shadow. Also, in order to reduce the mass and size of the optical elements, some vignetting was allowed at the C1 and C4 lenses. Figure 6 shows the Zemax estimate of the effects of the central obscuration, corrector shadow and lens vignetting on the system throughput with field radius.

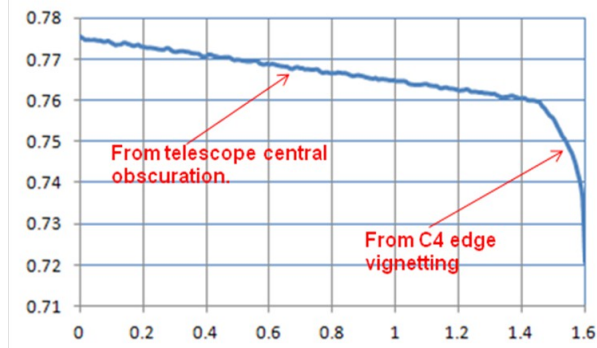

Figure 6. Throughput versus field radius (degrees) for the DESI corrector. 


\subsection{Focal plane}

Fiber injection efficiency is improved by minimizing deviation of the notional chief ray from the local fiber normal. Figure 7 shows the Echo22 chief ray deviation as a function of radial focal plate coordinates. The top panel shows the chief ray and the surface normal. These are subtracted to generate the central panel. On average, the chief ray deviation is less than $0.5^{\circ}$, and less than $1.0^{\circ}$ for all field positions. This suggests that the fiber normals may simply be mounted to follow the local normal of the focal plate. Finally, the lower panel shows the chief ray normal in red, and the surface normal in blue, to avoid ambiguity in the sign of the chief ray deviation.
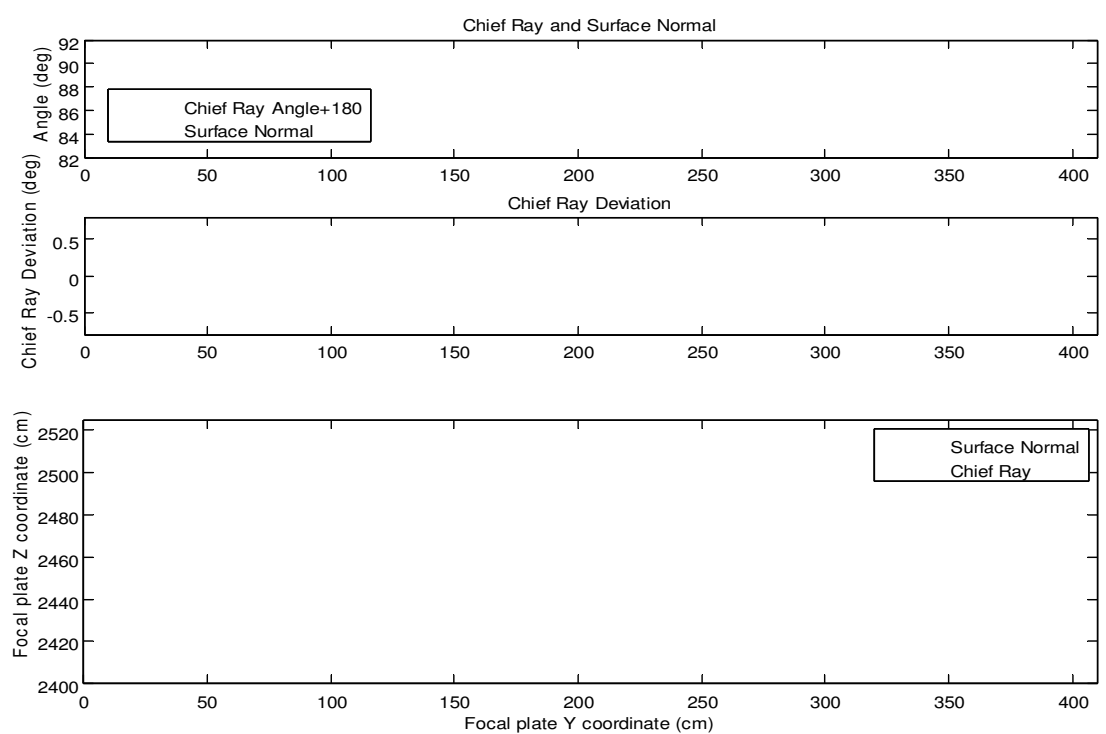

Figure 7. Relative orientation of chief ray and local focal surface normal. ( Note - the chief ray is notional for an obscured, prime focus configuration. Although the chief ray may be traced, it is blocked in reality by the prime focus corrector).

\section{LENS FABRICATION TOLERANCES}

Tolerances have been developed for the individual lenses of the corrector, and are shown in Table 4.

Table 4. Lens manufacturing tolerances

\begin{tabular}{|c|c|c|c|c|c|c|c|c|}
\hline $\begin{array}{c}\text { Lens } \\
\text { Surface }\end{array}$ & $\begin{array}{c}\text { ROC } \\
\text { tolerance } \\
\pm \mathrm{mm}\end{array}$ & $\begin{array}{l}\text { Thickness } \\
\text { tolerance } \\
\pm \mathrm{mm}\end{array}$ & $\begin{array}{c}\text { Wedge } \\
\mu \mathrm{m} / \text { diameter }\end{array}$ & $\begin{array}{l}\text { LSF figure error } \\
\text { P-V waves at } \\
\text { 633nm }\end{array}$ & $\begin{array}{l}\text { Surface } \\
\text { Slope error } \\
\text { RMS urad }\end{array}$ & $\begin{array}{c}\text { MSF/HSF } \\
\text { cutoff } \\
\text { pitch } \\
\text { mm } \\
\end{array}$ & $\begin{array}{c}\text { HSF } \\
\text { figure } \\
\text { error } \\
\text { RMS nm } \\
\end{array}$ & $\begin{array}{l}\text { HSF to } \\
\text { Roughness } \\
\text { cutoff mm }\end{array}$ \\
\hline C1_1 & 1 & 0.3 & 200 & 1 & 1 & 50 & 6 & 1 \\
\hline C1_2 & 3 & & & 1 & 1 & 45 & 6 & 1 \\
\hline C2_1 & 10 & 0.1 & 150 & 1 & 5 & 30 & 6 & 1 \\
\hline C2_2 & 0.5 & & & 1 & 2 & 25 & 6 & 1 \\
\hline ADC1_1 & 10 & 0.3 & 200 & 1 & 2 & 25 & 6 & 1 \\
\hline ADC1_2 & 2 & & & 1 & 2 & 20 & 6 & 1 \\
\hline ADC2_1 & 2 & 0.3 & 200 & 1 & 2 & 20 & 6 & 1 \\
\hline ADC2_2 & 1 & & & 1 & 2 & 20 & 6 & 1 \\
\hline C3_1 & 2 & 0.15 & 200 & 2 & 5 & 20 & 6 & 1 \\
\hline C3_2 & 1 & & & 2 & 2 & 20 & 6 & 1 \\
\hline C4_1 & 1 & 0.05 & 200 & 2 & 3 & 10 & 6 & 1 \\
\hline C4_2 & 10 & & & 2 & 3 & 10 & 6 & 1 \\
\hline
\end{tabular}


These Low Spatial Frequency (LSF) errors are defined as low-order Zernike terms and are defined over full diameter of the optic. For the purpose of slope errors, there is no distinction between LSF and mid-spatial-frequency (MSF) errors. Surface slope errors apply from largest scale (diameter) of optic to MSF/HSF cut-off pitch. High Spatial Frequency (HSF) figure error applies from the MSF/HSF cut-off pitch to the $1 \mathrm{~mm}$ scale, beyond which surfaces errors are considered roughness for which the specification is 20nm RMS. The tolerances are derived from performance budgets that allocate tolerances to lens radii error, thickness errors, surface figure errors (including high- and mid-spatial polishing errors), and others. Some effort has been put into specifying the figure errors, since their effects upon optical performance are subtle and not universally understood.

\section{CURRENT STATUS}

The corrector optical design underwent a successful external review in October 2013. A request for proposal (RFP) for the glass blanks for the fused silica lenses was issued in March 2014. Quotes were received from 4 companies and these at time of writing are undergoing assessment. An RFP for the polishing and coating of the fused silica lenses was issued in April 2014 with due date of for responses of the end of June 2014.

\section{REFERENCES}

1. Levi, M. et al., "The DESI Experiment , a white paper for Snowmass 2013”, ArXiv e-prints [Internet]arXiv:1308.0847, (2013)

2. Jacoby, G. H., Liang, Ming, Vaughnn, David., Reed, Richard., Armandroff, Taft., “A New Wide-Field Corrector for the Kitt Peak Mayall 3.8-m Telescope”, Proc. SPIE 3355, p721-734, (1998).

3. Kent, S. et al., "Preliminary optical design for a 2.2 degree diameter prime focus corrector for the Blanco 3.8 meter telescope", Proc. SPIE 6269 (2006).

4. Komiyama, Y. et al., ”HyperSuprime:imaging optics”, Proc. SPIE 6269 (2006). 